\title{
Cranial nerve contrast using nerve- specific fluorophores improved by paired-agent imaging with indocyanine green as a control agent
}

\author{
Veronica C. Torres \\ Victoria D. Vuong \\ Todd Wilson \\ Joshua Wewel \\ Richard W. Byrne \\ Kenneth M. Tichauer
}




\title{
Cranial nerve contrast using nerve-specific fluorophores improved by paired-agent imaging with indocyanine green as a control agent
}

\author{
Veronica C. Torres, ${ }^{a}$ Victoria D. Vuong, ${ }^{b}$ Todd Wilson, ${ }^{a}$ Joshua Wewel, ${ }^{c}$ Richard W. Byrne, ${ }^{c}$ and \\ Kenneth M. Tichauer ${ }^{a, *}$ \\ alllinois Institute of Technology, Department of Biomedical Engineering, Chicago, Illinois, United States \\ ${ }^{\mathrm{b}}$ Rush University Medical Center, Rush Medical College, Chicago, Illinois, United States \\ ${ }^{\mathrm{c}}$ Rush University Medical Center, Department of Neurosurgery, Chicago, Illinois, United States
}

\begin{abstract}
Nerve preservation during surgery is critical because damage can result in significant morbidity. This remains a challenge especially for skull base surgeries where cranial nerves (CNs) are involved because visualization and access are particularly poor in that location. We present a paired-agent imaging method to enhance identification of CNs using nerve-specific fluorophores. Two myelin-targeting imaging agents were evaluated, Oxazine 4 and Rhodamine 800, and coadministered with a control agent, indocyanine green, either intravenously or topically in rats. Fluorescence imaging was performed on excised brains ex vivo, and nerve contrast was evaluated via paired-agent ratiometric data analysis. Although contrast was improved among all experimental groups using paired-agent imaging compared to conventional, solely targeted imaging, Oxazine 4 applied directly exhibited the greatest enhancement, with a minimum 3 times improvement in CNs delineation. This work highlights the importance of accounting for nonspecific signal of targeted agents, and demonstrates that paired-agent imaging is one method capable of doing so. Although staining, rinsing, and imaging protocols need to be optimized, these findings serve as a demonstration for the potential use of paired-agent imaging to improve contrast of CNs, and consequently, surgical outcome. ๑ 2017 Society of Photo-Optical Instrumentation Engineers (SPIE) [DOI: 10.1117/1.JBO.22.9.096012]
\end{abstract}

Keywords: nerve labeling; cranial nerves; fluorescence; kinetic modeling.

Paper 170027RR received Jan. 12, 2017; accepted for publication Sep. 1, 2017; published online Sep. $23,2017$.

\section{Introduction}

Nerve damage is a major cause of surgery-associated morbidity that can lead to long-term loss of function, sensation, and chronic pain. ${ }^{1-9}$ It is especially crucial to preserve cranial nerves $(\mathrm{CN})$ because of their vital functions, and in skull base surgeries, this is a major challenge. ${ }^{10-12}$ With a limited field-of-view (FOV) and close proximity of critical structures in these surgeries, nerve visualization is difficult. Consequently, accidental nerve damage is still a common outcome despite preservation techniques and advances in surgical equipment; and recurrence of skull base tumors remains high because of incomplete resection resulting from risk of damaging these structures. ${ }^{10}$ The three most common tumors of the skull base are meningiomas, schwannomas, and pituitary adenomas. ${ }^{10,13}$ Posterior fossa meningiomas for instance, comprise $20 \%$ of all intracranial tumors and are associated with several CNs. ${ }^{14,15}$ Of the schwannomas, vestibular schwannoma in particular is directly involved with $\mathrm{CN}$ VII and VIII and represents $10 \%$ of all primary brain tumors. ${ }^{16-19}$ Therefore, it is of paramount importance that steps are taken to minimize the risk of iatrogenic $\mathrm{CN}$ damage.

Fluorescence image-guided surgery has emerged as a powerful tool for improving assessment of the surgical field and distinguishing between healthy and diseased tissue..$^{20-23}$ Recently, Whitney et al. $^{4-6}$ developed fluorescently labeled peptides (F-NP41) that target the connective tissue of nerves and they were able to demonstrate improved functional outcome of facial nerve branches after surgery in mice. Gibbs-Strauss et al. ${ }^{2,3}$ investigated the relationship between the structure and nervetargeting ability of fluorophores and identified three classes of small molecules that demonstrated preferential binding to nerves, of which distyrylbenzene (DSB) derivatives were the most promising. Investigators from GE global research synthesized DSBs, such as GE3082 and GE3111, which demonstrated an affinity for myelin basic protein, a major constituent of the myelin sheath surrounding nerves. ${ }^{7-9}$ Park et al. ${ }^{1}$ recently examined a variety of nerve-labeling fluorophores, and found Oxazine 4 to be the best contrast agent that most closely met the criteria of an ideal nerve contrast agent. A commonality in these studies is that nerve highlighting was done systemically and predominantly evaluated large nerves, such as the sciatic nerve and brachial plexus. In addition, much of the work was focused on contrast agent development. However, a recurring finding was nonspecific uptake and retention of agent in surrounding tissue, where accumulation was strongest in adipose tissue because of the lipophilic nature of the contrast agents.

In order to address this problem, a paired-agent imaging approach can be applied. Recent work has proven the utility of this method for eliminating unwanted signal caused by nonspecific uptake. ${ }^{24}$ With this technique, a second, untargeted control imaging agent is coadministered with the targeted agent, and 
the result is a more accurate quantification of receptor expression..$^{25,26}$ In this study, the goal was to develop pairedagent imaging methods for nerve contrast for the first time using existing promising nerve-labeling agents. As such, two approaches for $\mathrm{CN}$ imaging were taken: (1) paired-agent imaging to account for nonspecific uptake and (2) direct application of imaging agents in order to reduce agent total body dose.

\section{Materials and Methods}

\subsection{Paired-Agent Model}

The development and validation of the paired-agent approaches for quantifying concentrations of targeted biomolecules in tissue have been extensively demonstrated in previous publications for both systemically delivered imaging agents ${ }^{25-33}$ and for direct administration (topical) applications. ${ }^{34-38}$ Briefly, paired-agent approaches require coadministration of a control, "untargeted" imaging agent with the targeted agent(s), signal from which is used to account for variability in targeted imaging agent delivery and nonspecific retention, such that the concentration of biomolecules targeted can be estimated. Based on compartment model approximation of imaging agent kinetics in tissues, the simplest method of estimating biomolecule concentration is through the following equation:

$B P=\frac{\text { Tar-Untar }}{\text { Untar }}$

where Tar is the measured concentration of targeted agent in a region of interest, Untar is the measured concentration of "untargeted" control agent, and $B P$ is the "binding potential," a parameter that is the product of the targeted imaging agent affinity (a constant), and the concentration of the targeted biomolecule. ${ }^{39}$ Essentially, the ratio equates to the proportion of how much targeted imaging agent is bound, normalized by how much agent is unbound; and the magnitude of this ratio is directly proportional to how abundant the targeted biomolecule is. Figure 1 illustrates the ideas of nonspecific retention of imaging agent in the tissue, and how differencing and normalization results in an estimation of targeted biomolecule concentration (which in this case is myelin basic protein).

\subsection{Animal Experiments}

Animal studies were performed in accordance with approved institutional protocols (IACUC \#2013-008). Fifteen male Fischer rats weighing 200 to $250 \mathrm{~g}$ were obtained from Envigo (Indianapolis, Indiana) and separated into three groups. Two targeted agents were used in this study, Oxazine 4 and Rhodamine 800 , both of which have demonstrated an affinity for myelin basic protein. ${ }^{1}$ In the first two groups, Oxazine 4 (Exciton, Dayton, Ohio) was used as the targeted imaging agent. Direct application of the fluorophore was conducted in the first group $(n=5)$, whereas intravenous administration was done in the second $(n=5)$. For the third group, the targeted agent, Rhodamine 800 (Sigma-Aldrich, St. Louis, Missouri), was directly applied to the specimen $(n=5)$. In all cases, indocyanine green (ICG) (Chem-Impex International, Wood Dale, Illinois) served as the control imaging agent. Targeted and control imaging agents were dissolved in solutions containing $10 \%$ dimethyl sulfoxide (Amresco, Solon, Ohio), 5\% Kolliphor EL

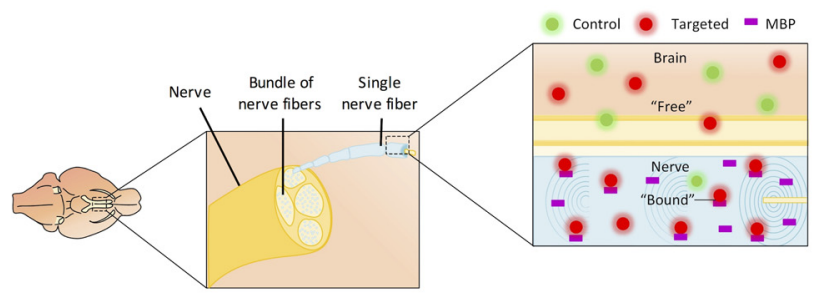

(a)
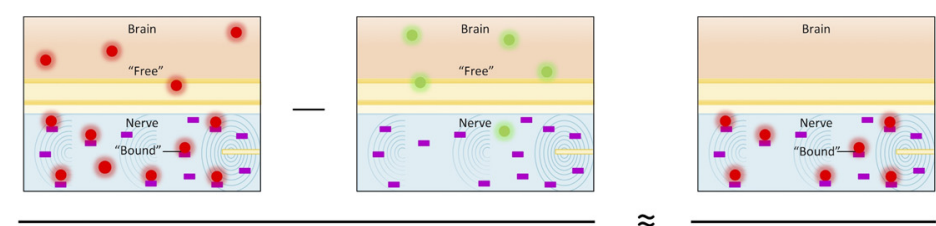

$\approx$
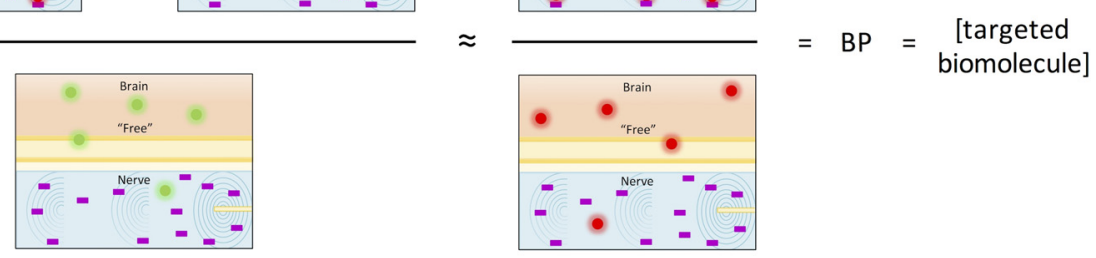

(b)

Fig. 1 Paired-agent model for quantification of binding in CNs. (a) Illustration of the distribution of targeted and control imaging agents in tissue. It is presumed that the control agent (green) is dispersed throughout one compartment, the "free" space; and the targeted agent (red) is dispersed throughout two compartments: the "free" space and the "bound" space. It is also assumed that both imaging agents can have nonspecific binding. (b) Illustration of the estimation of BP based on targeted and control imaging agent uptake. If it is assumed that the concentration of control agent in the region of interest is similar to the concentration of unbound targeted agent, and that the adiabatic approximation holds, BP can be approximated, which is directly proportional to receptor concentration. MBP: myelin basic protein, BP: binding potential. 
(Sigma-Aldrich, Saint Louis, Missouri), 65\% fetal bovine serum (FBS) (GE Healthcare Life Science, Logan, Utah), and 20\% HEPES buffer (ThermoFisher Scientific, Waltham, Massachusetts), at $2 \mathrm{mM}$ and $2 \mu \mathrm{M}$ concentrations, respectively. ICG is known to bind with high affinity to serum proteins. ${ }^{40,41}$ By adding FBS to the solution, the ICG was prebound in solution to minimize nonspecific binding in tissue.

For direct application studies [Fig. 2(a)], rats were sacrificed and brains dissected, keeping as many CNs intact as possible. Control images were taken to account for background fluorescence. Fresh brain tissues were stained ex vivo for $10 \mathrm{~min}$ with $500 \mu \mathrm{L}$ of the fluorescent cocktail. This was followed by three rinse steps of phosphate-buffered saline (PBS): three flushes per rinse. Each flush consisted of pipetting $1 \mathrm{~mL}$ of PBS onto the brain surface, and each rinse was completed with the removal of any residual PBS. Fluorescence images were then acquired.

Nerve staining via systemic administration was achieved via tail or penile vein injection [Fig. 2(b)]. Rats were given the fluorescent cocktail at a dose of $1 \mu \mathrm{mol} / 250 \mathrm{~g}$. Previous studies using Oxazine 4 to highlight nerves demonstrated optimal contrast $4 \mathrm{~h}$ after a single intravenous injection; ${ }^{1}$ thus, 4-h postinjection, the rats were euthanized, brains dissected, and imaged.

\subsection{Fluorescence Imaging Systems}

A custom-built multiwavelength fluorescence imaging system was used to acquire Oxazine 4, as well as ICG images, to allow for application of the paired-agent approach. The system consisted of a WheeLED wavelength-switchable light source (Mightex Systems, Pleasanton, California). Emitted light was passed through a filter wheel with bandpass filters designed to match each light channel. The light was then split into two liquid light guides to provide even illumination of the surgical field from two sides. A Basler ace NIR monochrome camera (Basler, Ahrensburg, Germany) with an 8-mm fixed focal length lens (Navitar, Rochester, New York) and appropriate emission filters were used to collect fluorescence images. All filters were obtained from Omega Optical (Brattleboro, Vermont) unless specified otherwise. Oxazine 4 fluorescence was acquired using a $617 \mathrm{~nm}$ LED filtered with a $615 \pm 5 \mathrm{~nm}$ bandpass excitation filter and a $655 \pm 25 \mathrm{~nm}$ bandpass emission filter. For ICG, a $780 \mathrm{~nm}$ light was filtered with a $785 \pm 12.5 \mathrm{~nm}$ bandpass excitation filter (Chroma Technology, Bellow Falls, Vermont), and the resulting emission fluorescence was collected with an $825 \mathrm{~nm}$ longpass filter (Edmund Optics, Barrington, New Jersey). White light images were acquired unfiltered. Total imaging time to acquire a targeted fluorescence, control fluorescence, and white light image was $50 \mathrm{~s}$; however, this was just a research-based system used for proof-of-concept, and a more advanced system could collect all images in video-rate if necessary. ${ }^{42}$

A Pearl Trilogy Imaging System (LI-COR Biosciences, Lincoln, Nebraska) was used to take white light, Rhodamine 800, and ICG fluorescence images. Acquisition of all three images took $38 \mathrm{~s}$. Rhodamine fluorescence was collected in the $700 \mathrm{~nm}$ channel, whereas ICG fluorescence was collected in the $800 \mathrm{~nm}$ channel.

\subsection{Image Analysis}

In order to compare performance of the different experimental groups, nerve contrast was quantified in two ways. First, nerve-

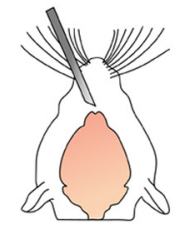

Dissect brain

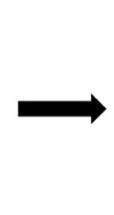

Image (background)

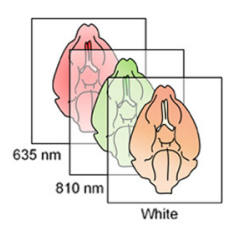

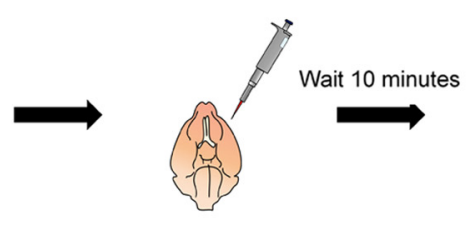

Stain with mixed solution of imaging agents

(a)

(a)

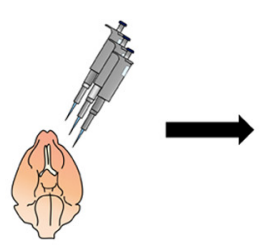

Rinse with PBS three times

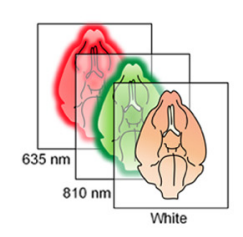

Image

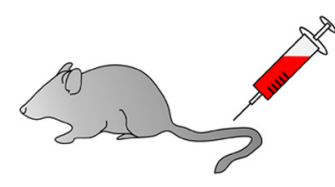

Inject mixed solution of imaging agents (b)

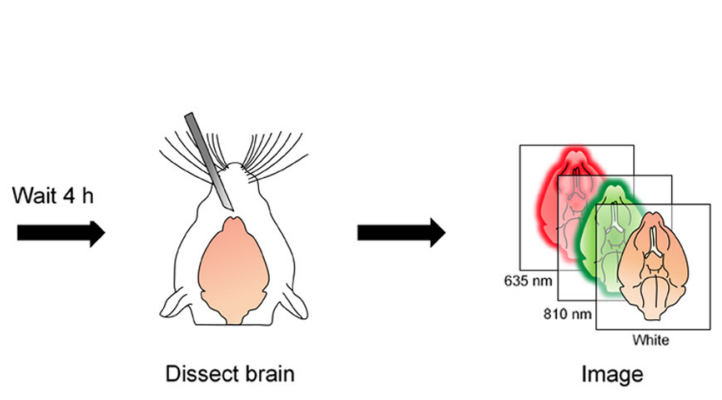

Image

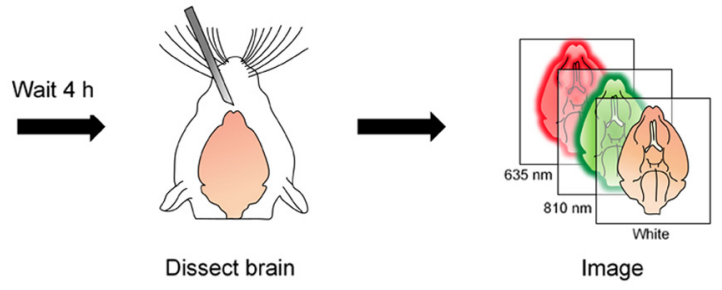

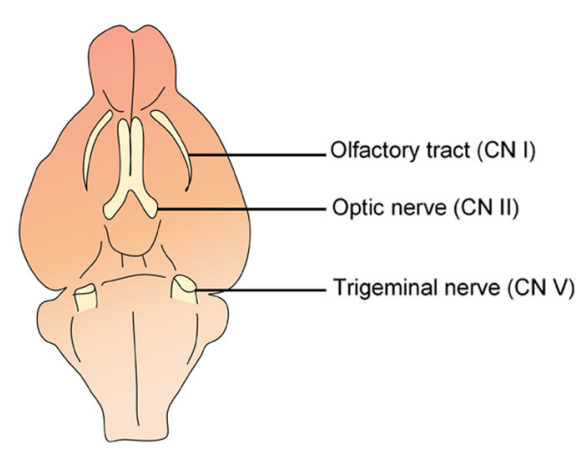

(c)

Fig. 2 Experimental procedure for paired-agent imaging of CNs. (a) Using the direct method of imaging agent application, staining was done ex vivo on brains from sacrificed rats. After obtaining background images in the targeted and control fluorescence channels, as well as white light, a mixture of targeted and control imaging agents was applied to the brains. This was allowed to stain for $10 \mathrm{~min}$, followed by three rinse steps. Fluorescence and white light imaging was then repeated. (b) For intravenous administration, the mixed solution of imaging agents was injected via the tail or penile vein. Four-h postinjection, brains were dissected from sacrificed rats, and imaging was done ex vivo. (c) Illustration of the ventral view of a rat brain. CNs used for analysis were the olfactory tract (CN I), optic nerve (CN II), and trigeminal nerve (CN V). 
to-brain ratio (NBR) was simply calculated as a signal-to-background ratio: average signal in a nerve region over the average signal in a brain region of similar size $\left(\mathrm{NBR}=S_{n} / S_{b}\right)$. The second measure used was contrast-to-noise ratio (CNR), which can be expressed as follows:

$\mathrm{CNR}=\frac{S_{n}-S_{b}}{\sigma_{b}}$,

where $S_{n}$ is the average signal in a nerve region, $S_{b}$ is the average signal in a brain region of similar size, and $\sigma_{b}$ is the standard deviation of the brain signal. In this study, three nerve regions, $\mathrm{CN}$ I, CN II, and CN V, were analyzed because they were most consistently preserved in the brain dissections [Fig. 2(c)]. Note: since this study was a proof-of-concept work comparing nerve contrast enhancement with paired-agent imaging and singleagent imaging, nerve regions were selected only from obvious nerves, and background regions were selected from brain tissue obviously devoid of CNs (as identified by a clinical neurosurgeon, author J.W.). Since the pairs of CN I and CN V appear as separate structures, nerve signal was measured as the average from both sides. To account for spatial variation, ratios were calculated as the average of four NBR or CNR values, each of which used a different brain region for $S_{b}$. The regions analyzed were in the left and right temporal lobes, hypothalamus, and pons.

For qualitative analysis, and to simulate the potential utility of nerve-specific imaging agents in conjunction with a control agent to highlight CNs, raw fluorescence data were thresholded and overlaid onto white light images. A logistic function ${ }^{43}$ was used to determine the optimum lower threshold that maximized nerve contrast for each individual image. A univariate color map where transparency increased as the lower threshold approached was applied to replicate how fluorescence might actually be visualized with overlay on a white light image for the surgeon. Furthermore, overlay of the white light image would provide surgeons with anatomical context, and the transparent to opaque color map avoids changing the native FOV.

\subsection{Simulation}

To test the utility of NBR and CNR as metrics for image quality, theoretical nerve images with different signals were simulated, and ratios were calculated using the equations above. Both images had a large and small nerve contained within a background. It was assumed that the theoretical images were acquired with a camera that was optimally exposed, and the first image had amplified signal compared to the second due to increased gain. Since image sensors measure signal as the number of photons detected over a given time, photon counting follows Poisson statistics with signal-dependent noise. Thus, Poisson noise was independently added to the signals (in nerve, ranging from $40 \%$ to $100 \%$ of the detector dynamic range; and in background, ranging from $7 \%$ to $33 \%$ of the detector dynamic range) from each simulated image using the function poissrnd() in MATLAB, at $1 \times$ and $2 \times$ simulated gain settings. On average, the second image had $\sim 7$ times more noise than the first. Since the truth in classifying nerve and background was known, sensitivity and specificity were calculated for both images using 50 different thresholds ranging from the minimum image intensity to the maximum. Sensitivity was calculated as the true positive rate (proportion of times nerves were correctly identified as nerves), and specificity as the true negative rate (proportion of times the background was correctly identified as such). A receiver-operating characteristic (ROC) curve was constructed by plotting the true positive rate (sensitivity) versus the false positive rate ( $1-$ specificity), and the area under the curve (AUC) was calculated. Corresponding NBR and CNR values were plotted against AUC values to compare ratios and their probability to accurately identify a pixel as nerve or background.

\subsection{Statistical Analysis}

All statistical analyses were performed using SPSS (IBM ${ }^{\circledR}$, Armonk, New York). A paired-samples $t$-test was used to compare contrast between single-agent and paired-agent imaging methods within each experimental group. Significance among the groups was evaluated with independent samples $t$-test and a Bonferroni correction to account for multiple comparisons. Analyses were done for each of the three CNs separately, and then averaged together. The level of statistical significance was set at $p<0.05$. Results are presented as mean \pm standard deviation unless otherwise noted.

\section{Results}

\subsection{Ex Vivo Nerve-Specific Fluorophore Cranial Nerve Staining in Rats}

Direct application of Oxazine 4 and Rhodamine 800 onto freshly excised rat brains was evaluated for nerve-specific fluorescence of CNs. Figure 3(a) shows representative images of control and targeted fluorescence uptake, as well as BP maps after applying the paired-agent model. Qualitatively, as expected, the control agent demonstrated similar uptake in both groups where fluorescence can be seen across the surface of the brain. Targeted fluorescence images of Oxazine 4 compared to Rhodamine 800, however, showed greater uptake in each of the nerve regions than in adjacent brain tissue located within the midbrain [Fig. 3(b)]. Direct application of Rhodamine 800 resulted in similar signal within CN I $(0.12 \pm 0.15)$ and the brain tissue $(0.13 \pm 0.15)$. Paired- and independent-samples $t$-tests indicated that there was no significant difference between signal from each nerve and the brain tissue, nor among test groups. Thus, neither of the agents could be quantitatively identified as the ideal candidate alone.

To better quantify the data, targeted fluorescence intensities from each region of interest were used to calculate NBR. Ratios were taken as the average among all nerve regions. Between groups, there was no significant difference when evaluating NBR with a single targeted agent, however, use of the paired-agent approach resulted in significant increases $(p<0.005)$ for both Oxazine 4 and Rhodamine 800 [Fig. 3(c)]. From inspection of their respective BP maps [Fig. 3(a)], nerves were much easier to locate with the application of paired-agent imaging using Oxazine 4 (NBR: $174.8 \pm 103.9$ ) as the targeted agent, as opposed to Rhodamine 800 (NBR: $71.1 \pm 88.3$ ) as the targeted agent. Contrast quantified by NBR was improved $61.2 \pm 31.0$ and $50.7 \pm 62.6$ times for Oxazine 4 direct and Rhodamine 800 direct groups, respectively [Fig. 3(c)].

Strong signal seen along the brainstem can be attributed to the presence of CNs as well. However, since it was difficult to preserve all of them consistently during the brain dissections, or track locations of specific CNs, they were not analyzed in this study. 


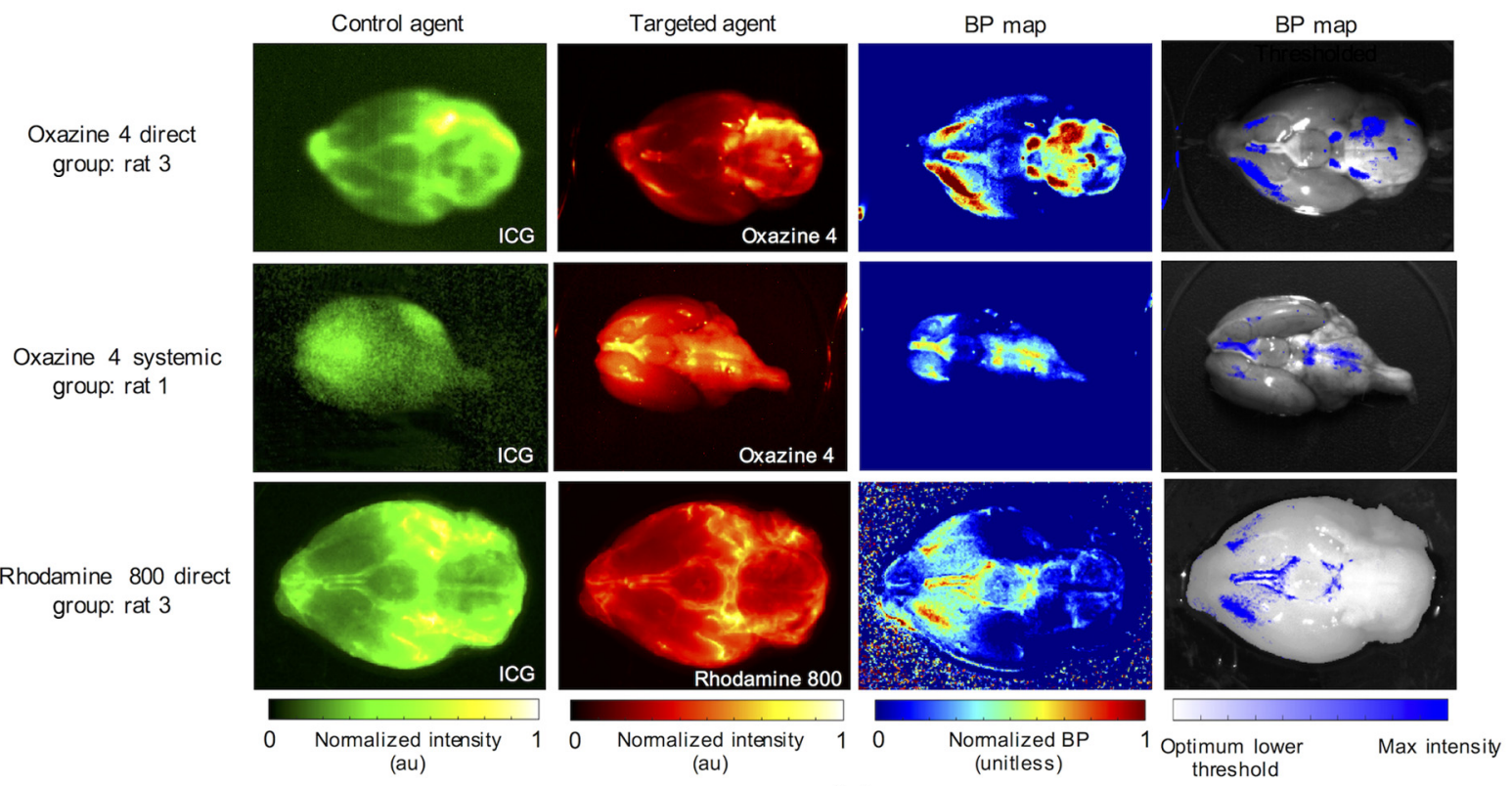

(a)

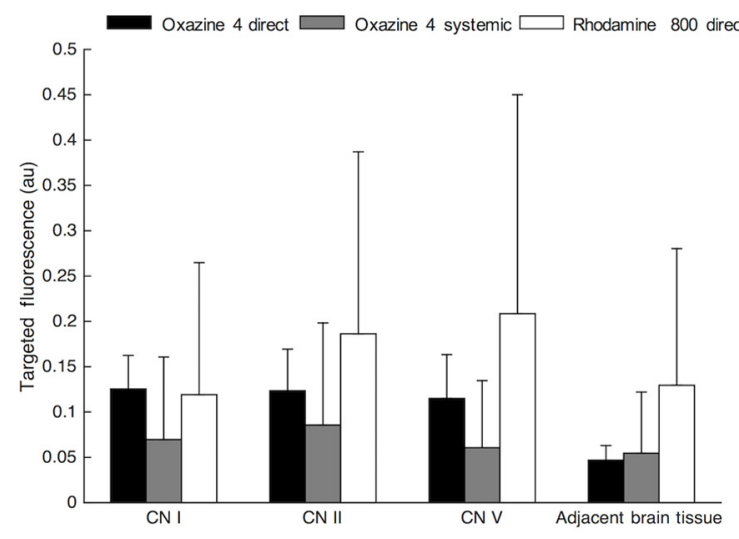

(b)

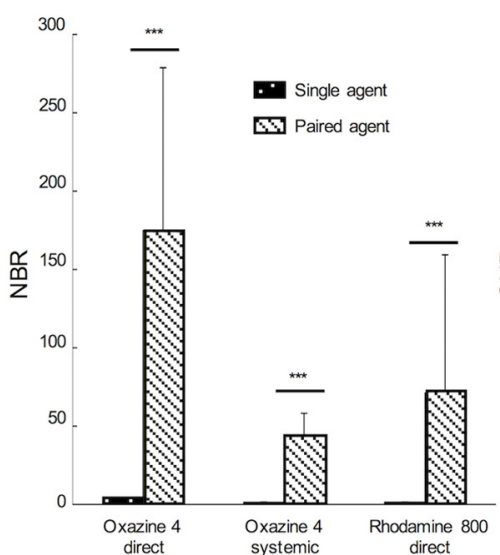

(c)

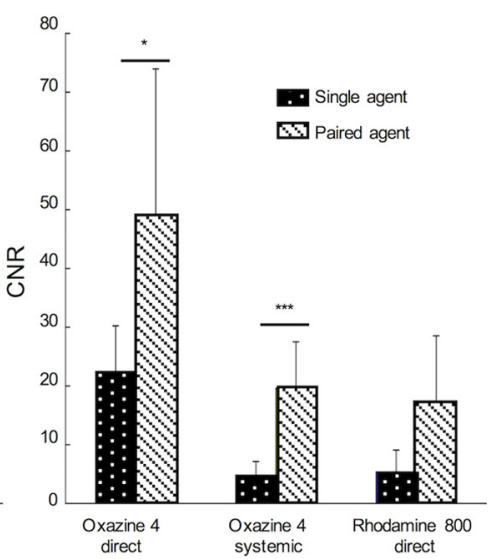

(d)

Fig. 3 (a) False-colored raw fluorescence images of ex vivo rat brains from the ventral view. Each row is representative of independent experiments for different experimental groups. First row: Oxazine 4 direct, second row: Oxazine 4 systemic, third row: Rhodamine 800 direct ( $n=5$ in each group). The first column presents residual fluorescence of the control agent, ICG, across the brain and nerve tissue. The second column displays targeted fluorescence uptake (either Oxazine 4 or Rhodamine 800 ) in the same brains. The third column illustrates BP maps, resulting from application of the paired-agent model. The fourth column shows thresholded BP maps overlaid onto white light images of the same brains. For each image of the last column, the maximum value was set to the maximum intensity of the color scale, and the minimum value was independently chosen as the optimum lower threshold to maximize nerve contrast. Units of fluorescence are arbitrary, and BP is a unitless value. Images from the Oxazine 4 groups were acquired using a custom-built imaging system (FOV: $14.4 \mathrm{~cm} \times 14.4 \mathrm{~cm}$ ), where ICG and Oxazine 4 images were collected at 785 and $655 \mathrm{~nm}$, respectively. In the third experimental group, using Rhodamine 800 , control images were taken in the $800 \mathrm{~nm}$ channel, whereas targeted images were captured in the $700 \mathrm{~nm}$ channel using a pearl trilogy imaging system (FOV: $11.2 \mathrm{~cm} \times 8.4 \mathrm{~cm}$ ). (b) Targeted fluorescence signal in nerve regions and a region of brain tissue located adjacent to nerves within the midbrain. (c) NBR and (d) CNR versus experimental group for both single- and paired-agent methods. Values are presented as the average of all three nerves evaluated. Results from all bar plots are the mean + standard deviation.

\subsection{In Vivo Oxazine 4 Cranial Nerve Staining in Rats}

In order to compare nerve specificity between direct application and systemic administration, in vivo nerve staining using an intravenously injected Oxazine 4/ICG mixture was conducted.
The second row of Fig. 3 displays a representative image of control and targeted fluorescence, in addition to BP maps, with rats euthanized $4 \mathrm{~h}$ after intravenous injection, prior to brain excision. Although there appeared to be some delineation of nerves in the Oxazine 4-targeted fluorescence image, there was also 
strong signal throughout the background (NBR: $1.4 \pm 0.1$ ), resulting in no statistically significant difference between the two regions [Figs. 3(a) and 3(b)]. Similar to the ex vivo results presented above, nerves were easier to identify relative to the brain when using the BP map images compared to the control or targeted fluorescent images alone. On average, contrast was improved $30.6 \pm 10.2$ times. Previous studies investigating the uptake of Oxazine 4 in the central nervous system of mice demonstrated no uptake in the brain; ${ }^{1}$ however, the same results were not obtained in this work, with brain signal being $3.0 \pm 2.2$ times higher than autofluorescence. Nonetheless, application of the paired-agent approach accounted for much of the nonspecific uptake. Little to no signal was observed using the control agent likely because of the large size of ICG molecules upon binding to plasma proteins $\mathrm{s}^{40,41}$ that prohibit it from crossing the blood-brain barrier (BBB) and blood-nerve barrier (BNB). Targeted agents were not affected by the BBB and BNB because they do not bind to large plasma proteins, and are inherently smaller molecules (Rhodamine 800: $495.95 \mathrm{~g} / \mathrm{mol}$, Oxazine 4: $395.84 \mathrm{~g} / \mathrm{mol})$ compared to ICG $(774.96 \mathrm{~g} / \mathrm{mol})$, the latter of which binds to proteins in blood (typically albumin; $66.5 \mathrm{kDa}$ ) that are over 100 times larger than Rhodamine 800 and Oxazine 4. In the case of direct application where ICG uptake could be visualized, size of the molecule was not a problem because the agent did not have to exit the vasculature.

Compared to the direct method of administration, nervespecific fluorescence signal from intravenous administration of Oxazine 4 was not as strong. Again, although the intensity of nerve signal was visually brighter in the targeted image of the systemic group, the rest of the brain was also correspondingly bright. The difference in contrast was more obvious when comparing the BP map images, where BP in nerve regions is stronger in the Oxazine 4 direct group.

\subsection{Quantification of Fluorescence Signal Using Contrast-to-Noise Ratio}

The CNR values provided significant differences between Oxazine 4 direct, and both Oxazine 4 systemic and Rhodamine 800 groups when using targeted fluorescence alone $(p<0.005)$. If paired-agent imaging was used, significance among the groups decreased $(p<0.05)$. Figure 5 illustrates this in more detail. Within groups, noteworthy increases in CNR were observed when comparing single-agent versus paired-agent imaging. The CNR improved $2.1 \pm 0.7$ times when applying Oxazine 4 directly; there was a $5.2 \pm 1.5$ time increase for intravenous administration of Oxazine 4; and CNR using direct application of Rhodamine 800 increased by a factor of $4.0 \pm 2.9$ [Fig. 3(d)].

Both NBR and CNR support the enhanced delineation of nerves in BP map images using the paired-agent approach as opposed to a single targeted agent. The relationships correlate well with what is depicted in the last three columns of Fig. 3(a).

However, it should be noted that the values obtained for NBR and CNR have rather drastic differences despite being measures of contrast for the same images. The NBR calculations reported values as high as 278 , whereas the maximum value returned for CNR was 74. Both ratios correspond to paired-agent imaging with Oxazine 4 applied directly; thus, such stark differences demonstrate the challenge of what metric best represents an image.

\subsection{Simulation to Demonstrate the Utility of Contrast-to-Noise Ratio as a Metric for Image Quality}

Theoretical images of a large nerve and smaller branch with varying levels of noise in the background are presented in Figs. 4(a) and 4(b). In the first image [Fig. 4(a)], both the large and smaller nerves were easily identified, with a NBR of 3. On the other hand, the same image with a higher NBR of 7.6 had a less distinguishable smaller nerve because of a noise level $\sim 7$ times higher [Fig. 4(b)]. Despite the suggested increase in contrast, resolution was clearly diminished in the second situation, where the smaller nerve was no longer clear and got lost in the background. This demonstrated the danger of relying on signal-to-background ratios as a quantitative measure of image quality because they do not account for noise. Alternatively, the CNR value was 3 times greater for the first image compared to the second. This difference more accurately reflected what was seen qualitatively, therefore suggesting that CNR is more informative as a single metric of image quality. This concept was further verified by plotting NBR and CNR versus AUC of the ROC [Fig. 4(c)]. For the simulated images, sensitivity and specificity were calculated for different thresholds, and an ROC curve was constructed. The plot illustrates wide variability of NBR values for single AUC values, whereas data points for CNR demonstrated a stronger correlation with AUC values and a consistent correlation approaching that of an ideal observer. This simulation further supports CNR as a more accurate quantitative representation of image quality in

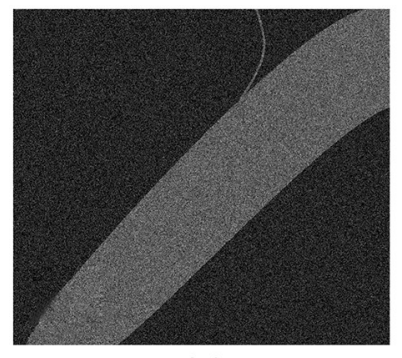

(a)

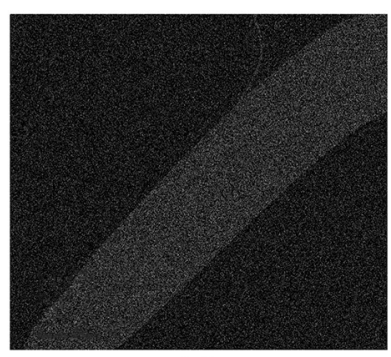

(b)

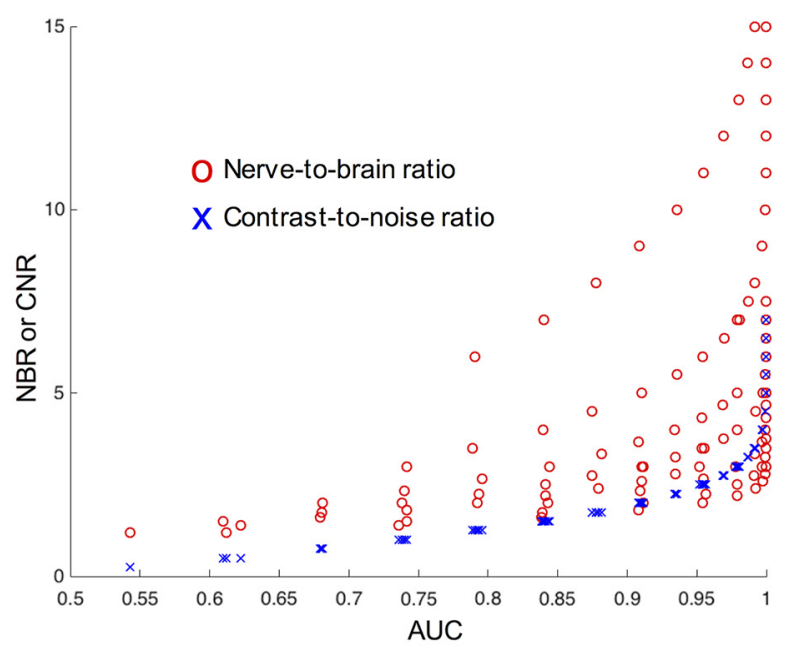

(c)
Fig. 4 Comparison of metrics to assess contrast from simulation. (a, b) Theoretical image of background tissue and two different-sized nerves with varied levels of noise. (c) Comparison of NBR and CNR against AUC of the ROC for the simulated situation of image (a). 
fluorescence-guided surgery compared to NBR or targeted fluorescence alone.

\subsection{Direct Application of Oxazine 4 Improves Contrast-to-Noise Ratio}

As discussed, CNR provided more meaningful information on image quality, and so a more in-depth analysis of the various groups was carried out using this metric. Figure 5 presents box plots of both single- and paired-agent nerve CNR for all three experimental groups. On average [Fig. 5(a)], nerve CNR was significantly greater in paired-agent imaging for Oxazine 4 direct and Oxazine 4 systemic groups $(p<0.05$ and $p<0.005$, respectively). The Rhodamine 800 direct group also showed an increase, although not statistically significant $(p>0.1$, NS). A similar pattern was revealed when looking at each nerve individually [Figs. 5(b)-5(d)], except for CNR of CN V for Oxazine 4 direct where there was no significant difference. The group that appeared to benefit most considerably from the paired-agent approach was the Oxazine 4 systemic group ( $p<0.005$ for average of all nerves). It demonstrated increases by factors of $7.4 \pm 4.5,5.2 \pm 2.5$, and $5.6 \pm 2.3$ for CN I, CN II, and CN V, respectively. The lack of significance in the Rhodamine 800 group can be attributed to the outliers. Nonetheless, these results support the benefits of paired-agent imaging.

Between groups, the box plots show rather consistent increases in CNR between the Oxazine 4 direct and Oxazine 4 systemic group, and Oxazine 4 direct and Rhodamine 800 direct group. This was evident for both single- and paired-agent imaging for the average of all nerves $(p<0.05)$. In single-agent CNR [Fig. 5(a), red dots], Oxazine 4 direct showed a $6.2 \pm 2.6$ time increase over Oxazine 4 systemic, and a $4.8 \pm 1.7$ time increase over Rhodamine 800 direct. Similarly, paired-agent CNR [Fig. 5(b), blue dots] for all nerves also displayed improvements with a $2.7 \pm 1.8$ time increase in Oxazine 4 direct over systemic, and a $3.3 \pm 2.1$ time increase over Rhodamine 800 .

\section{Discussion}

Nerve preservation during surgery remains a challenge owing to the fact that nerve can be difficult to identify. Current nervesparing techniques rely on anatomical landmarks or response to electrical stimulation; however, neither method is sufficiently reliable. ${ }^{7}$ This is of even greater consequence for skull base surgeries where CNs are involved. ${ }^{10-12}$ Although fluorescent

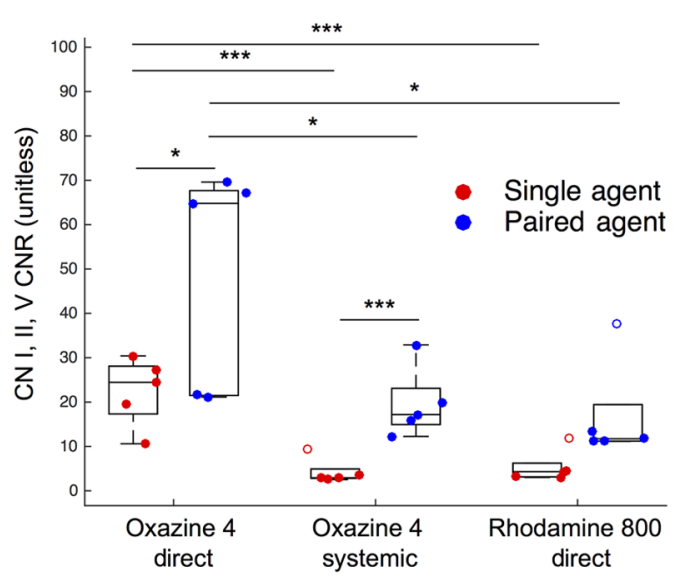

(a)

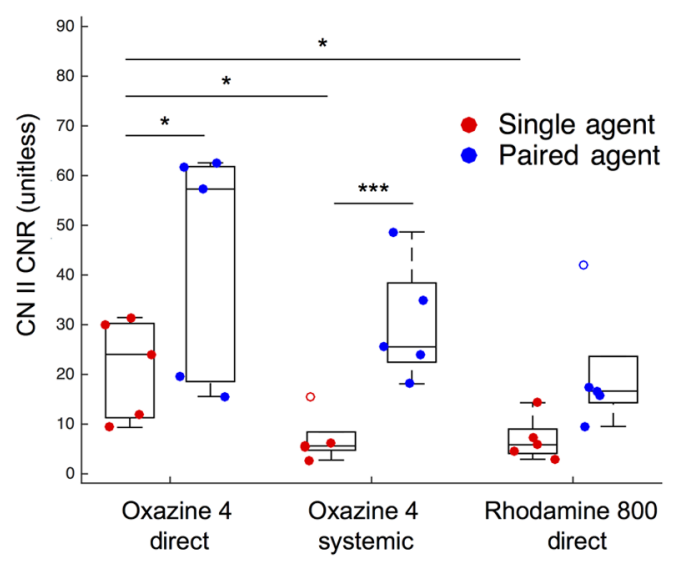

(c)

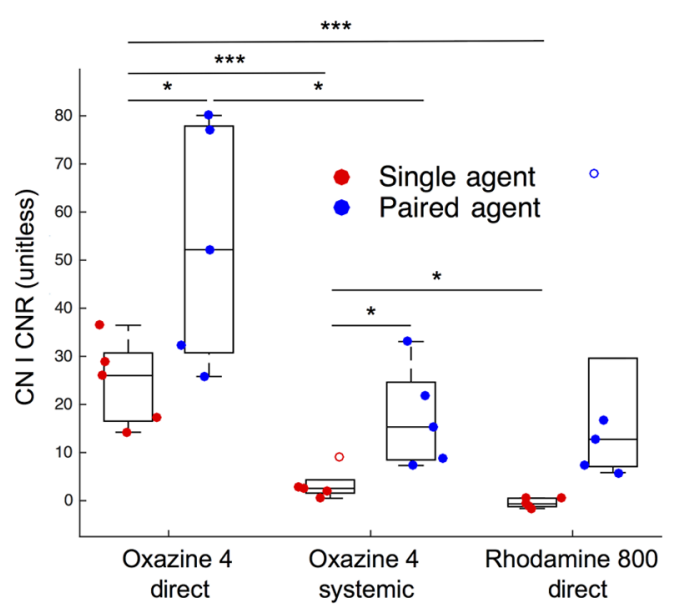

(b)

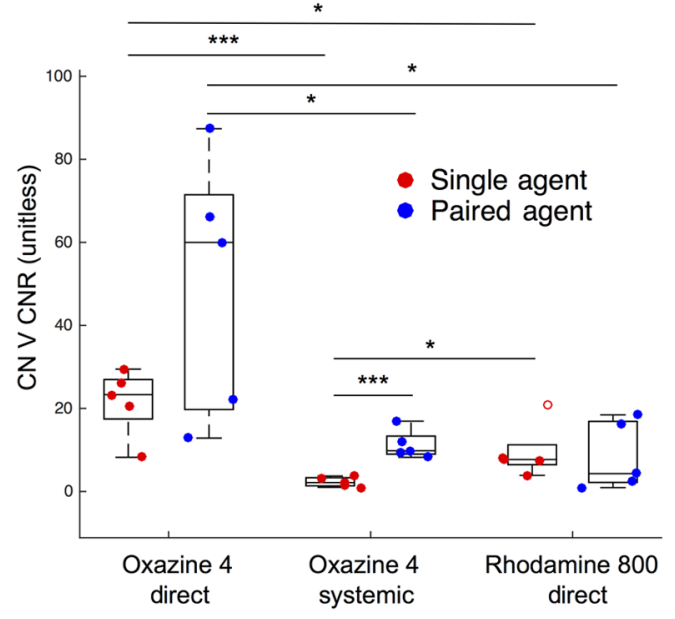

(d)

Fig. 5 Comparison of CNR versus targeted agent type and method of application between single- and paired-agent imaging for (a) the average of all nerves analyzed and (b) CN I, (c) CN II, and (d) CN V, independently. Each data point represents nerve CNR from one mouse. ${ }^{\star} p<0.05,{ }^{\star \star} p<0.01$, ${ }^{* * *} p<0.005$. 
molecules have been developed/identified to successfully target nerves, they also exhibit strong uptake in skin, muscle, and adipose tissue. In response to this challenge, this study proposed a paired-agent imaging approach that employs a second control agent to account for nonspecific uptake of the nerve-targeted agent. To test the paired-agent method, candidate nerve-specific fluorophores, along with a control agent were applied to rat brains, and performance was evaluated based on nerve discrimination, predominantly through the CNR metric.

Based on the results, there were two noteworthy findings in this study. First, was the validation of the benefit of paired-agent imaging. For both Oxazine 4 and Rhodamine 800 applied directly, and Oxazine 4 intravenously, the ability to resolve nerves was greatly improved with the paired-agent method compared to conventional targeted fluorescence alone. This was true qualitatively from simple inspection of the fluorescence images [Fig. 3(a)], but it was also corroborated by quantitative measures of NBR and CNR [Figs. 3(c), 3(d), and 5]. On average, for all nerves and groups, paired-agent imaging lent a $47.5 \pm 15.5$ time improvement over single-agent imaging for NBR, and a $3.8 \pm$ 1.6 time increase for CNR. As mentioned above, one of the major drawbacks of many of the nerve-specific fluorophores is the strong uptake in surrounding tissue. With the addition of a control agent, this unwanted signal was suppressed. Another limitation of single targeted agent imaging is the variability in optical properties for different tissues (e.g., higher scattering in myelin compared to brain tissue).$^{44,45}$ This can be accounted for with reflectance imaging optical property correction approaches; ${ }^{46,47}$ however, the paired-agent method has been demonstrated to be able to correct for optical property effects. ${ }^{48}$

The second major conclusion of this work was that Oxazine 4 applied directly provided superior nerve contrast over the same agent administered intravenously, or Rhodamine 800 also applied directly. Again, this is illustrated in Fig. 3(a), as well as supported with quantification of NBR and CNR [Figs. 3(c), 3(d), and 5]. On average for all nerves, direct application of Oxazine 4 improved NBR over the other groups by a factor of $1.9 \pm 0.3$ when using a single agent, and 4.6 \pm 3.6 after adding a control agent. Similarly, CNR was improved in the Oxazine 4 direct group compared to the Oxazine 4 systemic and Rhodamine 800 direct group on average, by a factor of $5.5 \pm 2.2$ for targeted fluorescence alone, and $3.0 \pm 2.0$ for paired-agent imaging. Moreover, this result is significant because it lends support for acceleration of FDA approval for such a contrast agent. With topical application, the imaging agent is applied directly to the region of interest. This means lower doses since the agent will not have to circulate throughout the body to reach its desired destination as required with intravenous administration. In addition, there is potential for reduced toxicity to the body because the imaging agent will only come into direct contact with the region of interest and its immediate surroundings.

Indeed, it can be argued that a second imaging agent increases the overall dose if it is assumed that the paired-agent method is equivalent to doubling the single-agent dose. To compare single-agent versus paired-agent $\mathrm{CNR}$ on an equivalent dose level, the results of this study demonstrating a 3.8 times contrast enhancement when using the paired-agent approach versus a single agent (i.e., $\mathrm{CNR}_{\text {paired }}=3.8 \mathrm{CNR}_{\text {single }}$ ) can be modified as follows. If the single-agent dose is doubled to match that of two agents, its CNR can be represented as $\mathrm{CNR}_{\text {single }, 2 \mathrm{x}}=\frac{2}{\sqrt{ } 2} \mathrm{CNR}_{\text {single }}$, where $\frac{2}{\sqrt{ } 2}$ accounts for the increased signal and associated Poisson noise from the greater dose. With this, paired-agent imaging would still lend a 2.7 time improvement in CNR.

It should also be noted that ICG is already FDA approved, and is being used clinically for video-angiography in cerebrovascular surgery in humans, where it has been shown to improve visualization and reduce surgical morbidity in aneurysm, brain arteriovenous malformations, extracranial-intracranial bypass, and CNS tumor surgeries. ${ }^{49-52}$ Therefore, it is both safe and useful for neurosurgical applications. This supports the use of ICG as a control imaging agent for the paired-agent approach, and in highlighting CNs.

Through simulations and quantitative analysis of the collected images, CNR was deemed a more informative metric of image quality when compared to NBR. In the simulation, values of CNR were more correlated than values of NBR with the ability to identify structures, thereby supporting CNR as a surrogate of ROC. That is, simulated images where nerves were easily identified had a higher CNR, and images where a smaller nerve branch visually disappeared had a lower CNR. This was not necessarily true for the relationship between NBR and nerve visualization. Furthermore, since CNR accounts for noise, the measure of contrast is less extreme than what a measure of NBR might provide. For instance, on average, Oxazine 4 applied directly provided a $47.5 \pm 15.5$ time increase in NBR over Oxazine 4 applied systemically, and Rhodamine 800 directly. The improvement in CNR, however, was subtler with a $3.8 \pm 1.6$ time increase.

This work served as a "proof-of-concept" investigation of the ability of previously studied nerve-targeting fluorophores to be applied directly rather than systemically as is conventionally done; and specifically, for CNs. The paired-agent approach proved to enhance nerve contrast further, however, the method does have some drawbacks. With the addition of another imaging agent, additional toxicology must be done, and FDA approval for two agents, which makes clinical translation more challenging. For the two targeted agents employed in this study, Oxazine 4 and Rhodamine 800 , there is no toxicology information available in the literature, so comprehensive toxicity studies would have to be carried out for a full optimization of dosing protocols. The control agent is already FDA-approved and its toxicity is well characterized, with no toxicity below $3 \mathrm{mM}$ for direct application ${ }^{53}$ (far greater than the $2 \mu \mathrm{M}$ used in this study). In addition, it is ideal for the two agents to have similar dynamics/properties. Other limitations of this study include consistency in the dissection of brains, and the stain and rinse protocol. The investigation of more, and smaller, nerves was prohibited by the difficulty of preserving all nerves when extracting the brains, and tracking their locations throughout the dissection and stain and rinse procedures. The use of larger animal models may help circumvent this challenge. Direct application of the imaging agents was completed by pipetting the mixed targeted and control agent solutions onto the surface of the brains, which may have resulted in nonuniform staining. Effects of this, however, were mitigated by paired-agent imaging because the control agent helps normalize delivery variability. Consequently, BP map images are not sensitive to distribution inconsistencies. Paired-agent imaging is therefore ideal in situations where there is nonuniform distribution of imaging agents. Nonetheless, a more consistent method of topical administration can be considered in future experiments to minimize delivery variability; perhaps a spray catheter could be used. 
Another limitation of the proposed protocol is that the $10 \mathrm{~min}$ stain time used in this study: while feasible in a clinical setting, it would not be ideal. In future work, a full characterization on how imaging agent dose, stain time, rinsing protocol, and imaging protocol affect $\mathrm{CN}$ contrast enhancement will be carried out. As the work presented in this paper is the first work investigating the feasibility of direct application of nerve-targeting agents to highlight $\mathrm{CNs}$, and significant improvements were observed in $\mathrm{CN}$ contrast enhancement with the $10 \mathrm{~min}$ protocol described, it is likely that further optimization of the protocol can significantly shorten imaging times.

In addition, future experiments will aim to more accurately mimic the clinical application of the proposed imaging method. This means skull base tumor animal models where contrast agents will be applied directly in vivo to the tumor region, and survival resection surgery will be performed.

\section{Conclusion}

In conclusion, the findings of this study demonstrate the potential for paired-agent imaging to improve contrast of CNs and eliminate nonspecific signal. Direct administration of Oxazine 4 proved to be superior in providing nerve contrast from surrounding brain tissue. As such, it is the candidate nerve-specific agent and method of administration for future studies. In addition to the topical application used in this work, with optimized stain and rinse procedures, common FDA approval obstacles associated with systemic injections can be overcome and clinical translation accelerated. With improved demarcation of $\mathrm{CNs}$, surgeons can perform more complete resections with the confidence that they are not endangering vital structures. Consequently, patient prognosis and quality of life can be improved.

\section{Disclosures}

The authors have no financial or other potential conflicts of interest to disclose.

\section{Acknowledgments}

The authors would like to acknowledge financial support provided by the Pritzker Fellowship in Biomedical Sciences and Engineering at Illinois Tech and the Rush University Medical College Dean's Office Summer Research Fellowship. The research was supported by the Rush University ArmourSchweppe Pilot Project Award and a Pritzker Institute of Biomedical Science and Engineering Seed Grant.

\section{References}

1. M. H. Park et al., "Prototype nerve-specific near-infrared fluorophores," Theranostics 4(8), 823-833 (2014).

2. S. L. Gibbs et al., "Structure-activity relationship of nerve-highlighting fluorophores," PLoS One 8(9), e73493 (2013).

3. S. L. Gibbs-Strauss et al., "Nerve-highlighting fluorescent contrast agents for image-guided surgery," Mol. Imaging 10(2), 91-101 (2011).

4. M. A. Whitney et al., "Fluorescent peptides highlight peripheral nerves during surgery in mice," Nat. Biotechnol. 29(4), 352-356 (2011).

5. A. P. Wu et al., "Improved facial nerve identification with novel fluorescently labeled probe," Laryngoscope 121(4), 805-810 (2011).

6. T. Hussain et al., "Fluorescently labeled peptide increases identification of degenerated facial nerve branches during surgery and improves functional outcome," PLoS One 10(3), e0119600 (2015).

7. V. E. Cotero et al., "Improved intraoperative visualization of nerves through a myelin-binding fluorophore and dual-mode laparoscopic imaging," PLoS One 10(6), e0130276 (2015).
8. D. C. Gray et al., "Dual-mode laparoscopic fluorescence image-guided surgery using a single camera," Biomed. Opt. Express 3(8), 1880-1890 (2012).

9. V. E. Cotero et al., "Intraoperative fluorescence imaging of peripheral and central nerves through a myelin-selective contrast agent," Mol. Imaging Biol. 14(6), 708-717 (2012).

10. J. N. Khanna, S. Natrajan, and J. Galinde, "Skull base tumors: a kaleidoscope of challenge," J. Neurol. Surg. Rep. 75(1), e11-e21 (2014).

11. C. C. Dong et al., "Intraoperative facial motor evoked potential monitoring with transcranial electrical stimulation during skull base surgery," Clin. Neurophysiol. 116(3), 588-596 (2005).

12. A. Morita, L. N. Sekhar, and D. C. Wright, "Current concepts in the management of tumors of the skull base," Cancer Control 5(2), 1-11 (1998).

13. P. M. Black, N. T. Zervas, and G. L. Candia, "Incidence and management of complications of transsphenoidal operation for pituitary adenomas," Neurosurgery 20(6), 920-924 (1987).

14. V. Velho et al., "Posterior fossa meningioma "our experience" in 64 cases," Asian J. Neurosurg. 7(3), 116-124 (2012).

15. U. Schick and W. Hassler, "Surgical management of tuberculum sellae meningiomas: involvement of the optic canal and visual outcome," J. Neurol. Neurosurg. Psychiatry 76(7), 977-983 (2005).

16. J. Betka et al., "Complications of microsurgery of vestibular schwannoma," BioMed Res. Int. 2014, 315952 (2014).

17. T. Sameshima et al., "Evaluation of variation in the course of the facial nerve, nerve adhesion to tumors, and postoperative facial palsy in acoustic neuroma," J. Neurol. Surg. B Skull Base 74(1), 39-43 (2013).

18. H. S. Xing et al., "Protection of facial nerves during acoustic neuroma surgery," Cell Biochem. Biophys. 72(1), 73-76 (2015).

19. J. Zhang et al., "Facial and cochlear nerve complications following microsurgical resection of vestibular schwannomas in a series of 221 cases," Med. Sci. Monit. 21, 1674-1678 (2015).

20. A. L. Vahrmeijer et al., "Image-guided cancer surgery using nearinfrared fluorescence," Nat. Rev. Clin. Oncol. 10(9), 507-518 (2013).

21. Q. T. Nguyen and R. Y. Tsien, "Fluorescence-guided surgery with live molecular navigation-a new cutting edge," Nat. Rev. Cancer 13(9), 653-662 (2013).

22. S. L. Gibbs, "Near infrared fluorescence for image-guided surgery," Quant. Imaging Med. Surg. 2(3), 177-187 (2012).

23. S. Gioux, H. S. Choi, and J. V. Frangioni, "Image-guided surgery using invisible near-infrared light: fundamentals of clinical translation," Mol. Imaging 9(5), 237-255 (2010).

24. K. M. Tichauer et al., "Quantitative in vivo cell-surface receptor imaging in oncology: kinetic modeling and paired-agent principles from nuclear medicine and optical imaging," Phys. Med. Biol. 60(14), R239-R269 (2015).

25. K. M. Tichauer et al., "Improved tumor contrast achieved by single time point dual-reporter fluorescence imaging," J. Biomed. Opt. 17(6), 066001 (2012).

26. K. M. Tichauer et al., "In vivo quantification of tumor receptor binding potential with dual-reporter molecular imaging," Mol. Imaging Biol. 14(5), 584-592 (2012).

27. K. S. Samkoe et al., "High vascular delivery of EGF, but low receptor binding rate is observed in AsPC-1 tumors as compared to normal pancreas," Mol. Imaging Biol. 14(4), 472-479 (2012).

28. K. M. Tichauer et al., "Advantages of a dual-tracer model over reference tissue models for binding potential measurement in tumors," Phys. Med. Biol. 57(20), 6647-6659 (2012).

29. B. W. Pogue et al., "Imaging targeted-agent binding in vivo with two probes," J. Biomed. Opt. 15(3), 030513 (2010).

30. K. M. Tichauer et al., "Accounting for pharmacokinetic differences in dual-tracer receptor density imaging," Phys. Med. Biol. 59(10), 2341-2351 (2014).

31. J. Baeten et al., "In vivo investigation of breast cancer progression by use of an internal control," Neoplasia 11(3), 220-227 (2009).

32. D. Pressman, E. D. Day, and M. Blau, "The use of paired labeling in the determination of tumor-localizing antibodies," Cancer Res. 17(9), $845-850$ (1957).

33. M. A. Bourdon et al., "Monoclonal antibody localization in subcutaneous and intracranial human glioma xenografts: paired-label and imaging analysis," Anticancer Res. 4(3), 133-140 (1984). 
34. L. Sinha et al., "Quantification of the binding potential of cell-surface receptors in fresh excised specimens via dual-probe modeling of SERS nanoparticles," Sci. Rep. 5, 8582 (2015).

35. J. T. Liu et al., "Quantifying cell-surface biomarker expression in thick tissues with ratiometric three-dimensional microscopy," Biophys. $J$. 96(6), 2405-2414 (2009).

36. Y. Wang et al., "Quantitative molecular phenotyping with topically applied SERS nanoparticles for intraoperative guidance of breast cancer lumpectomy," Sci. Rep. 6, 21242 (2016).

37. Y. W. Wang et al., "Rapid ratiometric biomarker detection with topically applied SERS nanoparticles," Technology 2(2), 118-132 (2014).

38. X. Xu et al., "Rinsing paired-agent model (RPAM) to quantify cellsurface receptor concentrations in topical staining applications of thick tissues," Phys. Med. Biol. 62(12), 5098-5113 (2017).

39. R. B. Innis et al., "Consensus nomenclature for in vivo imaging of reversibly binding radioligands," J. Cereb. Blood Flow Metab. 27(9), 1533-1539 (2007).

40. C. A. Davies-Venn et al., "Albumin-binding domain conjugate for near-infrared fluorescence lymphatic imaging," Mol. Imaging Biol. 14(3), 301-314 (2012).

41. G. R. Cherrick et al., "Indocyanine green: observations on its physical properties, plasma decay, and hepatic extraction," J. Clin. Invest. 39, 592-600 (1960).

42. S. L. Troyan et al., "The FLARE intraoperative near-infrared fluorescence imaging system: a first-in-human clinical trial in breast cancer sentinel lymph node mapping," Ann. Surg. Oncol. 16(10), 2943-2952 (2009).

43. J. T. Elliott et al., "Review of fluorescence guided surgery visualization and overlay techniques," Biomed. Opt. Express 6(10), 3765-3782 (2015).

44. J. F. Beek et al., "In vitro double-integrating-sphere optical properties of tissues between 630 and 1064 nm," Phys. Med. Biol. 42(11), 2255-2261 (1997).

45. A. N. Yaroslavsky et al., "Optical properties of selected native and coagulated human brain tissues in vitro in the visible and near infrared spectral range," Phys. Med. Biol. 47(12), 2059-2073 (2002).

46. D. Wirth et al., "Identifying brain neoplasms using dye-enhanced multimodal confocal imaging," J. Biomed. Opt. 17(2), 026012 (2012).

47. A. Kim et al., "Topographic mapping of subsurface fluorescent structures in tissue using multiwavelength excitation," J. Biomed. Opt. 15(6), 066026 (2010).

48. S. C. Kanick et al., "Pixel-based absorption correction for dual-tracer fluorescence imaging of receptor binding potential," Biomed. Opt. Express 5(10), 3280-3291 (2014).

49. P. Ferroli et al., "Application of intraoperative indocyanine green angiography for CNS tumors: results on the first 100 cases," Acta Neurochir. Suppl. 109, 251-257 (2011).

50. M. Hojo et al., "Usefulness of tumor blood flow imaging by intraoperative indocyanine green videoangiography in hemangioblastoma surgery," World Neurosurg. 82(3-4), e495-e501 (2014).
51. S. W. Hwang et al., "Intraoperative use of indocyanine green fluorescence videography for resection of a spinal cord hemangioblastoma," Op. Neurosurg. 67(3 Suppl. Operative), ons300-ons303 (2010).

52. S. Balamurugan et al., "Intra operative indocyanine green videoangiography in cerebrovascular surgery: an overview with review of literature," Asian J. Neurosurg. 6(2), 88-93 (2011).

53. R. Alford et al., "Toxicity of organic fluorophores used in molecular imaging: literature review," Mol. Imaging 8(6), 341-354 (2009).

Veronica C. Torres received her BS degree in biomedical engineering with a focus in neural engineering from Illinois Institute of Technology, Chicago, Illinois, in 2015. She is currently a Pritzker fellow working toward her $\mathrm{PhD}$ in biomedical engineering at the same institution. Her research interests include molecular imaging of cancer and biomedical optics.

Victoria D. Vuong received her BS degree in biochemistry and BA degree in neuroscience from the University of Southern California in 2011. She then received her ScM degree in reproductive and cancer biology from Johns Hopkins Bloomberg School of Public Health in 2013. Currently, she is a third year medical student at Rush Medical College. She plans to pursue postgraduate training in a neurosurgical residency following graduation.

Todd Wilson is currently at the Illinois Institute of Technology, pursuing a coterminal BS degree in biomedical engineering and master of imaging and sensing. $\mathrm{He}$ is interested in continuing research in biomedical optics and cancer treatment.

Joshua Wewel, MD, received his medical degree from the University of Nebraska College of Medicine, Omaha, Nebraska. He is currently a fifth year neurosurgical resident at Rush University Medical Center in Chicago, Illinois.

Richard W. Byrne, MD, is a neurosurgeon at Rush University Medical Center who has been in practice for over 19 years. He received his medical degree from Northwestern University Feinberg School of Medicine and completed his neurosurgery residency at Rush University. He specializes in surgical treatment for epilepsy, skull base and brain tumor surgery, pituitary surgery, trigeminal neuralgia and radiosurgery. His research areas include epileptogenesis, cortical mapping, brain tumor biology and tumor fluorescence.

Kenneth M. Tichauer has been an assistant professor in the Department of Biomedical Engineering, Illinois Institute of Technology, Chicago, Illinois, USA, since 2013. He has been awarded a CIHR postdoctoral fellowship and a CAREER grant from the NSF. His work is further funded by the Nayar Prize at IIT. His laboratory focuses on kinetic modeling in molecular imaging, predominantly in biomedical optics and cancer. 